\title{
Die Gegenwart ist grässlich
}

\section{Eine Schweizer Tagung erörtert das Verhältnis von Zeitdiagnosen und Wissenschaft}

\section{Daniel Deplazes}

Pädagogische Hochschule FHNw, Allgemeine und Historische Pädagogik, Obere Sternengasse 7, $\mathrm{CH}-4502$ Solothurn

d.deplazes@unibas.ch

Risiko-, Wissens- oder Beschleunigungsgesellschaft? Wie auch immer die Zeitdiagnose ausfällt, sie ist pessimistisch. Wie aussichtsreich ist eine erziehungswissenschaftliche Auseinandersetzung mit Zeitdiagnosen bei derart düsteren Perspektiven? Klassischerweise - und nicht erst seit Rousseau - ist die gegenwärtige Gesellschaft immer schlecht und die Lösung besteht darin, sich ihrem schändlichen Einfluss zu entziehen. Daher erstaunt es kaum, dass sich die Bildungsforschenden für die von Anne Bosche (Zürich), Patrick Bühler (Solothurn) und Michael Geiss (Zürich) organisierte Tagung »Bildungsforschung und Zeitdiagnose« vom 31. August bis 1. September 2017 in ein altertümliches Schloss zurückzogen. Das imposante Bauwerk auf der idyllischen Halbinsel Au, am Zürichsee gelegen, suggeriert schon Abgeschiedenheit, weil es erst nach einem kurzen Spaziergang durch Wald, an Wiesen und Teich vorbei zu erreichen ist und dabei unmittelbar an eine >Pädagogische Provinz $<$ erinnert. Trotz den weltabgewandten Vorzeichen scheuten die Vortragenden, die aus unterschiedlichen methodischen Forschungsrichtungen stammen, wie etwa quantitative, qualitative, historische oder philosophische Herangehensweisen, die produktive Auseinandersetzung mit der Welt nicht. Im Gegenteil zeugten die intensiven, fordernden und nicht zuletzt anregenden Diskussionen über die Funktion von Zeitdiagnosen und deren Verhältnis zur Bildungsforschung von einem unerschütterlichen >Interesse an der Welt<. Der zu erwartende Methodenstreit fiel marginal aus, während die Frage, was eine Zeitdiagnose genau sein solle oder eben nicht sein könne, im Tagungsverlauf zunehmend intensiver diskutiert wurde.

Einen Definitionsvorschlag lieferte gleich zu Beginn Heinz-Elmar Tenorth (Berlin) mit der These, dass Zeitdiagnosen eine »spezifische eigene Argumentationsart« mit wiedererkennbaren Merkmalen darstellten. Eine der entscheidenden Eigenheiten - wie sich auch im Verlauf der Tagung immer wieder 
herauskristallisierte - stelle die »Handlungsaufforderung « zur vermeintlichen Lösung der diagnostizierten Krise dar. Die zeitdiagnostische Rhetorik, welche Tenorth an historischen Texten etwa von Spranger festmachte, orientiere sich an einem »basalen Leitbegriff«, womit die Diagnose zwangsläufig unterkomplex ausfallen müsse. Sie schematisiere Zeit, so Tenorth, indem sie die Vergangenheit normativiere, die Gegenwart als verachtenswert beschreibe und die Zukunft positiv besetze. Der Diagnostiker schätze die aktuelle Zeit als die »Schlimmste« ein. Da sich eine solche Gegenwartseinschätzung jeweils mit der »ganzen Welt« beschäftige, könne die historische Bildungsforschung keine Zeitdiagnosen liefern, da sie sich schon aus methodologischen Gründen vernünftigerweise lediglich mit einem Ausschnitt der Welt beschäftige, pauschale Aussagen ablehne und Reformempfehlungen vermeide.

Der von Tenorth beschriebenen Andersartigkeit von Zeitdiagnosen und wissenschaftlicher Forschung hielt Norbert Ricken (Bochum) entgegen, Gegenwartsbestimmungen könnten trotz ihrer Problematik und Einseitigkeit unter Umständen doch produktiven Anregungswert für die Reflexion der eigenen Gesellschaft bieten. Die prekäre Situation der »Beobachtung zweiter Ordnung « (Luhmann), in der sich der_die Zeitdiagnostiker_in zwangsläufig befindet, dürfe jedoch ebenso wenig unterschlagen werden, wie die schwierige zeitdiagnostische »Ganzheits- und Therapieannahme«, auf deren Basis der_die Diagnostiker_in den Weg aus der Krise verspreche. Ricken plädierte daher für die kritische wissenschaftliche Analyse des unterschiedlichen Umgangs mit Zeitdiagnosen, wobei insbesondere die pädagogische Perspektive für die gesellschaftliche Reproduktion von Ungleichheit relevant sei. Auf die dabei anzutreffende Problematik der Unverfügbarkeit von >Gegenwart<, welche sich lediglich retrospektiv und somit historisch bestimmen lasse, wies Marcus Emmerich (Tübingen) hin: Was »jetzt« passiere, sei »unklar«, womit die Möglichkeit, überhaupt eine Zeitdiagnose zu stellen, grundsätzlich in Frage gestellt ist. Das Verhältnis zwischen dem Erziehungssystem und dem sozialen Konstrukt >Gesellschaft< funktioniere systemtheoretisch als ein Verhältnis von innen und außen, dem der_die Zeitdiagnostiker_in einen Kausalplan unterstelle, wie das Exempel der $>$ Bildungsexpansion`zeige. Soziologische Theorien würden als interdisziplinäre Teile der Erziehungswissenschaft dazu verhelfen, den jeweiligen Wandel sowie dessen Phänomene theoretisch und empirisch aufzuschließen, wobei Handlungs- und Kommunikationstheorien besonders bedeutsam seien. Die Frage, wie die Wissenschaft mit derlei Diagnosen zusammenhänge, wurde im Plenum kontrovers debattiert. Während einige Anwesende Zeitdiagnosen als für die Forschung unerheblich abfertigten, beschrieben sie andere als deren Ausgangspunkt. 
Die These des Zeitdiagnostikers_der Zeitdiagnostikerin als »Dramaqueen«, welche bereits aufgrund dieser dramaturgischen Rolle keinen Anspruch auf Wahrheit und Wissenschaftlichkeit haben könne, stellte Roland Reichenbach (Zürich) zur Diskussion. Zeitdiagnosen seien unterhaltsam und könnten unter Umständen Debatten entfachen, aber mehr nicht. Im »Zeitalter des Narzissmus« stehe die persönliche Einzigartigkeit im Zentrum jeder Rhetorik, womit die Befürchtung snormal $\mathrm{zu}$ sein unhaltbar werde und in wenig ergiebige, überempfindliche Diskurse münde. »Ich als X« sei die paradoxe, konstruierte Selbstbeschreibung, welche für alternative Erklärungsmuster der Welt blind sei und somit vom wesentlichen >inequality problem<, der finanziellen Ungleichheit, ablenke. Die simplifizierten Lösungen in Zeitdiagnosen erinnerten an die Psychiatrie: Der »komplexen Diagnose« folge die einfache Frage nach der Dosis des Medikaments.

Genau diese Sehnsucht in Zeitdiagnosen nach einfachen Lösungen, diesmal in Form einer tatsächlich verabreichten Pille, veranschaulichte Nicole Vidal (Freiburg) am Beispiel der medizinischen Diagnose ADHS. Das »Schwierige Kind « sei jedoch keine Zeitdiagnose, da gleiche oder sogar weitergehende Symptombeschreibungen seit Beginn des 20. Jahrhunderts existieren. Vielmehr sei der Umgang der jeweiligen Zeit mit dem »Schwierigen Kind« als Zeitdiagnose zu verstehen. Heute sei die Inklusion des hyperkinetischen Kindes in der Schule zum Leitmotiv geworden, was die praktische Pädagogik an ihre Grenzen bringe. Trotz ausbleibendem Nachweis habe sich in den 199oer-Jahren die Ansicht durchgesetzt, dass die Symptome einem »biologischen Bedingungssystem « unterlägen, was die Behandlung mit Medikamenten weiter beflügelt habe: die »Ritalin-Nation « als Zeitdiagnose einer realen Praxis mit ärztlich verschriebenem Heilmittel. Weder Eltern, Lehrer_innen noch Ärzte_Ärztinnen würden jedoch die zweifelhaften, mehrheitlich auf schulischen Unterricht gemünzten, Diagnoseinstrumente anzweifeln. Das vordergründige Ziel, das sstörende Kind « mittels Medikamenten wieder >funktionsfähig` zu machen und zu integrieren, bleibe unreflektiert. Dabei werde suggeriert, dass mit der Anwesenheit >aller S Schüler_innen in der Klasse, von störenden bis zu hochbegabten, das Inklusionsziel erfüllt sei. Ob aber so dem Inklusionsgedanken entsprochen werde, erscheint fragwürdig.

Wie schwammig der Begriff >Inklusion< ist, zeigte Justin J.W. Powell (Luxemburg) in einer komparatistischen Studie zur inklusiven Bildung. Bereits die empirische Erfassung von Inklusion mit dem messbaren Item der >Anwesenheit< von Kindern mit Förderbedarf im Schulzimmer unterschlage die Eigenheiten und historischen Bedingtheiten der Bildungssysteme der untersuchten Länder. Powell verwies auf die weltweit steigenden Inklusionsraten der Bildungssysteme bei gleichzeitig schwer zu überblickender Vielfalt 
der praktizierten Inklusionsformen im Graubereich zwischen Exklusion und Inklusion. Die Einseitigkeit einer Zeitdiagnose könne somit unter Umständen mittels quantitativer Forschung relativiert oder zumindest der »Status Quo« kritisiert werden.

Wie brüchig dabei jedoch die Konzepte und Theorien etwa von >Large Scale Assessements< anmuten, rief Anke Grotlüschen (Hamburg) in Erinnerung. Die heutigen, populären Zeitdiagnosen empirischer Messungen des politischen Selbstwirksamkeitsempfindens seien zunehmend problematisch, nicht zuletzt aufgrund der hierfür teilweise ungeeigneten und in die Jahre gekommenen $>$ Rational Choice-Theorie<, welche sich hinter den Items verberge. Zudem sei die Prämisse, wonach mehr politische Anteilnahme Demokratie befördere, aufgrund der jüngst vermehrt auftretenden zweifelhaften politischen Gruppierungen selbst zur Disposition zu stellen. Die Heterogenität in Bezug auf die politische Informiertheit der Politikabstinenten_abstinentinnen sowie der Wähler_innen lasse darauf schließen, dass Labels wie »Politikverdrossene« nicht länger haltbar seien. Die Politik fordere daher zu Unrecht aufgrund vermeintlich valider Studienergebnisse mehr "politische Bildung« für Randgruppen, um die unhaltbare Zeitdiagnose mittels derart einfacher Lösungen zu entschärfen. Dabei übersehe sie jedoch, dass sozial benachteiligte Populationen möglicherweise gute Gründe dafür hätten, sich nicht am politischen Prozess zu beteiligen und es ihnen unter Umständen keineswegs an politischer Bildung mangle.

Wie einmal gestellte Bildungsdiagnosen zu politischen Selbstläufern werden können, scheint nicht lediglich ein Problem aktueller Gesellschaftsbeobachtungen zu sein, sondern lässt sich historisch immer wieder feststellen. Welche Funktion etwa eine Zeitdiagnose wie die »Bildungskatastrophe« oder der »Fachkräftemangel« der 196oer- und 1970er-Jahre für die Bildungspolitik zeitigten, legte Philipp Eigenmann (Zürich) am Beispiel des Kantons Zürich dar. Dem damaligen Zeitdiagnostiker hätten zunehmend statistisch erfasste Entwicklungen, wie etwa die Zuwachsraten der Anzahl der Schüler_innen, als Grundlage für düstere Zukunftsprognosen gedient. Empirische Daten aus der Ökonomie zum angenommenen >Leistungsdefizit`sowie aus der Soziologie zum vermeintlichen `Gerechtigkeitsdefizit`hätten zur Zunahme des Einflusses dieser Disziplinen auf die Erziehungswissenschaft beigetragen und gleichzeitig der Bildungspolitik - und nicht nur ihr - die Narrative für ihr Handeln geliefert. Die »Orientierung an empirischen Fachexperten« sei mittels der Einrichtung einer »Bildungsplanungsstelle «, welche mit einem Soziologen besetzt wurde, institutionalisiert worden. Wie sich an Eigenmanns exemplarischer Untersuchung zeigt, sind Zeitdiagnosen unter Umständen mehr als Rhetorik. Offensichtlich beeinflussen sie die Praxis besonders dann, wenn sie die 
Ableitung für eine politische Reform aufgrund >harter Daten< immanent gleich mitliefern und somit Handlungsdruck erzeugen.

In eine ähnliche Richtung ging der Beitrag von Michael Geiss (Zürich), der die Auswirkungen der Zeitdiagnose der »Informationsgesellschaft« der 1970er-Jahre untersuchte. Im Licht des technologischen Fortschrittes habe der Zeitdiagnostiker damals Arbeitsplätze in Gefahr gesehen und als heilsversprechende Antwort auf eine ungewisse Zukunft das Konzept des >Lebenslangen Lernens angepriesen. Seit den 1980er-Jahren seien die finanziellen Anstrengungen vielerlei Staaten für die Behebung des vermeintlichen »Defizits im Technologiewesen « in Form der Lancierung von Förderungsprogrammen wie etwa ERASMUS kontinuierlich angestiegen. Unter dem Deckmantel, das Technologieproblem zu bearbeiten, hätten sich immer mehr Interessensgruppen um den zu verteilenden forschungsökonomisch bedeutsamen >Kuchen< versammelt. Diskurse wie das >Lebenslange Lernen $<$ hätten sich in diesem Prozess allmählich verselbstständigt und schließlich von der Technologiefrage abgekoppelt.

Wie aktuelle Zeitdiagnosen eine Praxis des `Lebenslangen Lernens $<$ beeinflussen können, veranschaulichte Bernd Käpplinger (Giessen) am Beispiel der Bildungsberatung für Erwachsene, welche eine Weiterbildung anstreben. Nicht zuletzt wirkten Zeitdiagnosen unmittelbar in Beratungsgesprächen, da die Klientel sich für die >richtige< Erwerbsbiografie zu entscheiden habe. Bei den von Käpplinger mittels Dokumentenanalyse und Tonbandaufnahmen untersuchten Beratungen zeige sich einerseits eine eingewobene Kontroll- und Steuerungsfunktion, zumal die finanzielle Unterstützung von Weiterbildungen den Besuch einer Bildungsberatung voraussetze und das Resultat, nämlich die Vergabe des »Bildungsschecks«, bereits im vornherein feststehe. Darum ergebe sich eine Antinomie zwischen einer Machtpraxis sensu Foucault und dem eigentlichen Wesen einer Beratung. Anderseits lasse sich, aufgrund des Zwangscharakters des Beratungsanlasses, eine »Eigenmächtigkeit der Praxis« feststellen. Beratende und ihr Klientel würden teilweise lediglich so agieren, als finde eine Beratung statt, um die formalen Bedingungen für die finanzielle Weiterbildungsunterstützung zu erfüllen. Was fehle, sei eine Metadiskussion zum Verhältnis von Zeitdiagnose und Beratung, was erst die Gefahren der Simplifizierung von Vergangenheit und Gegenwart auch für Beratungsgespräche problematisieren würde.

Den Zeitdiagnosen über Zeitdiagnosen im Schloss Au fehlte es an diesen beiden Tagen zumindest kaum an Debatten auf der Metaebene. Die Klärung von Verhältnissen wie etwa Theorie/Empirie, Zeitdiagnose/Soziologie, Zeitdiagnose/Wissenschaft standen im Zentrum der Diskussionen und obwohl Zeitdiagnosen geradezu zu zerbröseln beginnen, je mehr man ihnen zu Leibe 
rückt, erhärtete sich dennoch der Verdacht, dass sich die wissenschaftliche Auseinandersetzung mit ihnen lohnt. Darüber hinaus zogen Theorie und Empirie für einmal konstruktiv meist am gleichen Strick und hatten streckenweise, wie Reichenbach an einer Stelle anmerkte, »das Heu auf der gleichen Bühne«. Pädagogen_Pädagoginnen mögen diesen Umstand wohl der harmonischen Abgeschiedenheit der Tagungsstätte zuschreiben. Die malerische $>$ Heimatmosphäre< verließen die Teilnehmenden zumindest ohne eine »Handlungsaufforderung « einzuklagen, wobei der Verdacht, selbst eine Zeitdiagnose geliefert zu haben, dennoch nicht ganz von der Hand zu weisen ist. 